\title{
Perfil clínico-epidemiológico de mulheres em situação de abortamento em uma maternidade pública de São Luís-MA
}

\author{
Clinical and epidemiological profile of women in abortion situation in a public \\ maternity of São Luís - MA
}

\author{
Roberto Destêrro ${ }^{1}$, Silva Moreira Lima ${ }^{1}$, Lilalea Gonçalves França ${ }^{2}$, Raquel Castro \\ Desterro Silva Moreira Lima ${ }^{3}$
}

Resumo: Introdução: O abortamento constitui um problema de saúde pública importante e representa uma mudança relevante na saúde física e psicológica da mulher.Metodologia: Trata-se de estudo descritivo, quantitativo de mulheres hospitalizadas por abortamento na Maternidade Estadual Benedito Leite, em São Luís do Maranhão, no ano de 2015. Foram analisadas 100 pacientes quanto aos aspectos socioeconômicos e causas de abortamento. Os dados foram analisados no software Excel e expressos em porcentagens e médias.Resultados: Houve predomínio da faixa etária entre 20 e 28 anos $(57,0 \%)$,da cor parda $(51,0 \%)$, em situação conjugal de união estável $(45,0 \%)$, da procedência de São Luís - MA $(81,0 \%)$, da escolaridade de ensino médio completo $(49,0 \%)$, a maioria atuando em algum tipo de trabalho remunerado $(42,0 \%)$ e com renda de 2 a 3 salários mínimos $(62,0 \%)$. A maioria foi de não fumantes $(100,0 \%)$, que não ingeriam bebidas alcoólicas $(89,0 \%)$, sem realização de consultas pré-natal $(61,0 \%)$, menarca de 13 anos ou mais $(62,0 \%)$, sexarca de 15 a 18 anos $(75,0 \%)$, com mais de um parceiro sexual $(72,0 \%)$, sem doenças prévias $(92,0 \%)$, sem abortamento prévio $(82,0 \%)$, tendo realizado aborto tipo espontâneo $(88,0 \%)$, sem causa referida (81,0\%), $100,0 \%$ fizeram curetagem e $82,0 \%$ abortaram até a $12^{\text {a }}$ semana de gestação. Conclusão: $O$ abortamento ocorreu em sua maioria em mulheres jovens, com condições socioeconômicas precárias, com poucos fatores de risco e mais frequentemente do tipo espontâneo.

Palavras-Chave: Aborto. Saúde da mulher.Gestação.

Abstract: Introduction: The abortion is a serious problem of public health and an important change in physical and psychological health of the woman. Methodology: A descriptive and quantitative study was made with hospitalized women because of abortion in Maternidade Estadual Benedito Leite, in São Luís, capital of the state of Maranhão, in 2015. One hundred patients were analyzed in socio-economic aspects and causes of abortion. Data were analyzed in Excel software and described in percentages and means. Results: There was a dominant amount of women between the ages of 20 and 28 years old $(57,0 \%)$, brown $(51,0 \%)$, in stable union $(45,0 \%)$, from São Luís - MA $(81,0 \%)$, with complete high school education $(49,0 \%)$, which had a paid work $(42,0 \%)$, and with an income of 2 or 3 minimal wages $(62,0 \%)$. The majority was non-smoking $(100,0 \%)$, non-alcohol drinker $(89,0 \%)$, with no pre-natal check-up $(61,0 \%)$, menarche at 13 years of age or more(62,0\%), first sexual intercourse between 15 and 18 years of age $(75,0 \%)$, with more than 1 sexual partner $(72,0 \%)$, no previous diseases $(92,0 \%)$, no previous abortions $(82,0 \%)$, having spontaneous abortion $(88,0 \%)$, with unknown cause $(81,0 \%), 100,0 \%$ were submitted to acurettage and $82,0 \%$ aborted before the $12^{\text {th }}$ pregnancy week. Conclusion: The abortion happened at it most in young women, with poor socioeconomic conditions, with few risk factors and more often spontaneous type.

Key-Words: Abortion. Woman's health. Pregnancy

1 - Graduado do Curso de Medicina - UNICEUMA

2- Docente - UNICEUMA

3 -Médica do Hospital Universitário/MA

Rev. Investig, Bioméd. São Luís 7:16-27. 2015 


\section{Introdução}

A Organização Mundial de Saúde (OMS) define o abortamento como a interrupção da gravidez antes da viabilidade do produto da concepção, correspondendo à perda do concepto até a $22^{a}$ semana completa ou, em caso de idade gestacional desconhecida, peso fetal inferior a $500 \mathrm{mg}$ ou medindo menos de $16 \mathrm{~cm}^{1}$.Pode ser classificado quanto ao tipo, sendo considerado espontâneo quando o organismo expulsa 0 feto, geralmente devido a problemas de saúde da mulher ou do feto ou provocado quando é feito intencionalmente pela utilização de processo abortivo externo, químico ou mecânico. Este último pode ainda ser classificado em duas outras formas, de acordo com a motivação da mulher em voluntário ou involuntário da gestante, e ser considerado legal ou ilegal perante a lei ${ }^{2}$.

No mundo todo, as leis quanto à legalização do aborto variam bastante. $\mathrm{Na}$ França é considerada legal a prática do aborto até a $12^{\text {a }}$ semana de gestação ou em situações que coloquem a vida da gestante em risco. Nos Estados Unidos, durante o $1^{\circ}$ trimestre de gestação o aborto é livre, por decisão da mãe aconselhada pelo médico; no segundo trimestre, o aborto é permitido, mas o estado poderia regulamentar 0 exercício deste direito visando proteger a saúde da mulher; e no terceiro trimestre os governos podem intervir no aborto, objetivando a proteção da criança ${ }^{3}$.
O Código Penal, em seu Artigo 128, diz que "Não se pune o aborto praticado por médico: I - Se não há outro meio de salvar a vida da gestante; II - Se a gravidez resulta de estupro e o aborto é precedido de consentimento da gestante ou, quando incapaz, de seu representante legal" 4 .

Os abortos podem ser classificados quanto à idade gestacional e quanto à periodicidade em abortamento precoce, tardio e habitual. É dito precoce quando ocorre até 12 semanas e tardio entre 13 e 22 semanas de gestação ${ }^{5}$. Pode ainda ser classificado em abortamento habitual, podendo ser definido quando ocorrer três abortamentos espontâneos consecutivos ${ }^{6}$.

A OMS estima que, no mundo todo, cerca de 500 mil mulheres morrem a cada ano de causas relacionadas à gestação, sendo $98 \%$ das mesmas em países em desenvolvimento, onde ainda $15 \%$ correspondem a complicações de aborto ${ }^{7}$.Nos anos 1990, no Brasil, o aborto induzido ficou entre a terceira e a quarta causa de mortalidade materna. A estimativa oficial da razão de morte materna é de $76 / 100.000^{8}$.

Desde 1980 registra-se a subnotificação das mortes por aborto, apontadas como as mais mal notificadas dentre as mortes maternas $^{9}$. Em 2002, uma pesquisa realizada em capitais brasileiras e no Distrito Federal evidenciou que $11,4 \%$ dos óbitos maternos foram devido a complicações de abortos ${ }^{10}$. Outros estudos mostram valores que chegam a mais de 1 milhão de abortos induzidos realizados no período de 1 ano, mostrando ainda 
que sua maioria ocorreu nas regiões do nordeste e sudeste do país. Esses valores podem ser ainda maiores devido ao alto número de mulheres que omitem terem induzido 0 aborto em pesquisas com perguntas diretas ${ }^{8}$.

A maioria dos abortamentos espontâneos ocorre dentro das primeiras 12 semanas de gestação. A morte do embrião ou feto quase sempre precede a expulsão espontânea, que normalmente é acompanhada de hemorragia. A esta situação se segue a necrose do tecido adjacente que estimula as contrações uterinas e consequentemente a expulsão. Um saco gestacional intacto é geralmente preenchido pelo líquido e pode ou não conter um embrião ou feto ${ }^{11}$.

São várias as causas que podem levar a um abortamento espontâneo. Dentre elas existem as anormalidades cromossômicas, que correspondem a $50 \%$ desses abortamentos; há também as desordens anatômicas como miomas, malformações uterinas; as doenças endócrinas incluindo hipotireoidismo e diabetes melittus insulino-dependente descompensado no momento da concepção; a síndrome dos ovários policísticos; os distúrbios imunológicos; e os processos infecciosos como a rubéola, o citomegalovírus, a malária, a toxoplasmose dentre outras $^{12}$.

Além da idade menor que 15 e maior que 35 anos e abortamentos anteriores, outros fatores de risco para o abortamento incluem o alcoolismo, tabagismo, peso materno $(\mathrm{IMC}<19$ ou > 30 $\mathrm{kg} / \mathrm{m}^{2}$ ), exposições a agentes químicos, físicos e biológicos nocivos, certas condições clínicas como hipertensão, diabetes e doenças infecciosas e a não realização de consultas pré-natal ${ }^{13}$.

Para realizar a retirada do conteúdo intrauterino existem métodos cirúrgicos e clínicos. Dentre os cirúrgicos existem a Aspiração Manual Intrauterina (AMIU), que é o procedimento escolhido em gestações menores que 12 semanas, assim como a aspiração a vácuo. Quando a idade gestacional for superior a 12 semanas ou quando não for possível realizar a AMIU, o método de escolha é a curetagem ${ }^{7}$. A microcesariana é um método menos usado reservado para quando não for possível se resolver por via vaginal $^{1}$.

Dentre os métodos clínicos, a perfusão venosa de ocitocina pode ser feita até a expulsão do feto, seguida de esvaziamento uterino cirúrgico ${ }^{7}$. Além desse, o uso de misoprostol pode ser utilizado para favorecer a expulsão fetal ${ }^{1}$.

O abortamento constitui um problema de saúde pública importante e representa uma mudança relevante na saúde física e psicológica da mulher. Desta forma, o presente estudo poderá contribuir para o conhecimento científico e possíveis melhorias na qualidade da atenção à saúde sexual e reprodutiva das mulheres, objetivando traçar o perfil clínicoepidemiológico do aborto e caracterizando as mulheres internadas por abortamento.

\section{Material e Métodos}

O projeto deste estudo foi enviado para apreciação pelo Comitê de Ética em Pesquisa (CEP) da 
DOI: https://doi.org/10.24863/rib.v7i1.16

Universidade CEUMA em julho de 2015, obtendo aprovação para sua execução em setembro do mesmo ano, com o parecer consubstanciado número 1.199.377.

Trata-se de um estudo descritivo e quantitativo realizado no período de outubro de 2015, na Maternidade Estadual Benedito Leite, em São Luís, Maranhão, que avaliou mulheres internadas por abortamento em faixa etária reprodutiva que se propuseram voluntariamente a fornecer dados necessários ao estudo. Foram excluídas as mulheres analfabetas e que optaram por não assinar 0 Termo de Consentimento Livre e Esclarecido (TCLE). Cem mulheres foram consideradas aptas a participar do estudo.

O estudo teve as seguintes variáveis: idade, cor, situação conjugal, procedência, grau de escolaridade, ocupação habitual, renda familiar, tabagismo, alcoolismo, consultas pré-natal, idade da menarca, idade da primeira relação sexual, existência de mais de um parceiro sexual, existência de doenças prévias e de abortamento prévio, tipo, causa e método de abortamento.

A idade foi expressa em anos (até 19 anos, de 20 a 28, de 29 a 37 e maior que 38 anos); a cor foi descrita em "branca", "parda", "preta" ou "amarela" de acordo com o referido pela paciente; a situação conjugal das pacientes foi classificada em "solteira", "casada", "divorciada", "viúva" e "união estável". A procedência foi classificada em "São Luís", "interior do Maranhão" e "outro estado".

O grau de escolaridade constou de "Ensino Fundamental incompleto", "Ensino Fundamental completo", "Ensino Médio incompleto", "Ensino Médio completo", "Ensino Superior incompleto" e "Ensino Superior completo". A ocupação habitual foi classificada em "Estudante", "Atividade do lar" e "Trabalho remunerado". A renda familiar foi expressa em salários mínimos (até 1, de 2 a 3, 4 ou mais ou não sabe).

O tabagismo foi avaliado apenas pela resposta "sim" ou "não" ao fumo; da mesma forma foi avaliado o alcoolismo, pela resposta "sim" ou "não". Quanto ao número de consultas pré-natal foi divido em grupos em Nenhuma, 1 a 3 , e 4 ou mais. A idade da menarca foi dada em anos, divididos em menor de 10, 11 a 12 e 13 anos ou mais. A idade da primeira relação sexual foi dada em anos em até 14, de 15 a 18 e mais de 18 anos. Foi interrogada a existência de um ou mais parceiro sexual, além da existência de doenças prévias e de abortamento prévio. A idade gestacional na data do abortamento foi classificada em menos de 12 semanas, mais de 12 semanas ou não referida.

Foi aplicado um formulário sobre os dados socioeconômicos e clínicos das mulheres em situação de abortamento. Inicialmente foi feito um estudo piloto com dez pacientes para avaliar a abrangência do instrumento de coleta. Os dados obtidos foram digitados em um banco de dados no software Excel.

O projeto foi submetido ao Comitê de Ética em Pesquisa da Universidade Ceuma (CEP) em atendimento às exigências da Resolução 466/2012 do Conselho Nacional de Saúde que norteia a pesquisa envolvendo seres 
humanos. Foi garantida a confidencialidade das fontes de informação, sem identificação de qualquer sujeito participante do estudo. Foi solicitada de todas as participantes a assinatura do termo de consentimento livre e esclarecido.

\section{Resultados}

A seguir serão apresentados os dados coletados de $1^{\circ}$ setembro a 31 de outubro de 2015, relativos a 100 mulheres em situação de abortamento internadas na Maternidade Estadual Benedito Leite, em São Luís, MA.

$\mathrm{Na}$ Tabela 1, podem ser observadas as principais características demográficas dessas pacientes - predominou a faixa etária de 20 a 28 anos (57,0\%), a cor parda $(51,0 \%)$, a situação conjugal de união estável $(45,0 \%)$ e a procedência de São Luís, capital do estado do Maranhão $(81,0 \%)$. Na Tabela 2 são apresentadas as principais características socioeconômicas das mulheres em situação de abortamento. A escolaridade referida pelas pacientes foi de $49,0 \%$ de Ensino Médio completo, seguida de $26,0 \%$ de Ensino Médio incompleto. A ocupação habitual das pacientes foi de $42,0 \%$ de trabalho remunerado, seguida de $39,0 \%$ de atividade do lar; e a renda familiar das pacientes, foi de $62,0 \%$ de 2 a 3 salários mínimos.

Tabela 1. Características demográficas das mulheres internadas por abortamento. São Luís, Maranhão, 2015.

\begin{tabular}{|c|c|c|}
\hline Variáveis & $\mathbf{n}$ & $\%$ \\
\hline \multicolumn{3}{|l|}{ Idade (anos) } \\
\hline Até 19 & 17 & 17,0 \\
\hline $20-28$ & 57 & 57,0 \\
\hline $29-37$ & 23 & 23,0 \\
\hline 38 ou mais & 3 & 3,0 \\
\hline Total & 100 & 100,0 \\
\hline \multicolumn{3}{|l|}{ Cor } \\
\hline Branca & 35 & 35,0 \\
\hline Parda & 51 & 51,0 \\
\hline Preta & 14 & 14,0 \\
\hline Amarela & 0 & 0,0 \\
\hline Total & 100 & 100,0 \\
\hline \multicolumn{3}{|l|}{ Situação conjugal } \\
\hline Solteira & 27 & 27,0 \\
\hline Casada & 28 & 28,0 \\
\hline Divorciada & 0 & 0,0 \\
\hline Viúva & 0 & 0,0 \\
\hline União estável & 45 & 45,0 \\
\hline Total & 100 & 100,0 \\
\hline \multicolumn{3}{|l|}{ Procedência } \\
\hline São Luís & 81 & 81,0 \\
\hline Interior do Maranhão & 19 & 19,0 \\
\hline Outro estado & 0 & 0,0 \\
\hline Total & 100 & 100,0 \\
\hline
\end{tabular}


Tabela 2. Características socioeconômicas das mulheres internadas por abortamento. São Luís, Maranhão, 2015.

\begin{tabular}{lrc}
\hline \multicolumn{1}{c}{ Variáveis } & $\mathbf{n}$ & $\%$ \\
\hline Escolaridade & & \\
Ensino Fundamental incompleto & 10 & 10,0 \\
Ensino Fundamental completo & 3 & 3,0 \\
Ensino Médio incompleto & 26 & 26,0 \\
Ensino Médio completo & 49 & 49,0 \\
Ensino Superior incompleto & 5 & 5,0 \\
Ensino Superior completo & 7 & 7,0 \\
Total & $\mathbf{1 0 0}$ & $\mathbf{1 0 0 , 0}$ \\
Ocupação habitual & & \\
Estudante & 19 & 19,0 \\
Atividade do lar & 39 & 39,0 \\
Trabalho remunerado & 42 & 42,0 \\
Total & $\mathbf{1 0 0}$ & $\mathbf{1 0 0 , 0}$ \\
Renda (salário mínimo) & & \\
Até 1 & 20 & 20,0 \\
2-3 & 62 & 62,0 \\
4 ou mais & 18 & 18,0 \\
Total & $\mathbf{1 0 0}$ & $\mathbf{1 0 0 , 0}$ \\
\hline
\end{tabular}

Com relação aos fatores de risco apresentados pelas mulheres em situação de abortamento, foi referido não fumar em $100,0 \%$ delas e não ingerir bebidas alcoólicas em $89,0 \%$. Sessenta e um por cento das pacientes referiram não ter feito nenhuma consulta pré-natal. A menarca dessas mulheres ocorreu em $62,0 \%$ na idade de 13 anos ou mais e a primeira relação sexual em $75,0 \%$ aconteceu nas idades de 15 a 18 anos. Setenta e dois por cento tiveram mais de um parceiro sexual. Poucas pacientes referiram ter doenças prévias como Mioma (3,0\%), Hipertensão (2,0\%) e
Ovários policísticos (2,0\%); a maioria $(92,0 \%)$ negou ter qualquer doença prévia ao abortamento. Oitenta e dois por cento das mulheres nunca passaram pela situação de abortamento antes (Tabela 3).

As características do abortamento sofrido pelas mulheres estudadas podem ser observadas na Tabela 4. O abortamento espontâneo aconteceu em 88,0\% das mulheres. As mesmas referiram causas variadas para 0 abortamento; entre elas $5,0 \%$ foi uso de misoprostol, 7,0\% ocasionadas por queda e $81,0 \%$ 
Tabela 3. Fatores de risco de mulheres internadas por abortamento. São Luís, Maranhão, 2015.

\begin{tabular}{|c|c|c|}
\hline Variáveis & $\mathbf{n}$ & $\%$ \\
\hline \multicolumn{3}{|l|}{ Fumo } \\
\hline Sim & 0 & 0,0 \\
\hline Não & 100 & 100,0 \\
\hline Total & 100 & 100,0 \\
\hline \multicolumn{3}{|l|}{ Álcool } \\
\hline $\operatorname{Sim}$ & 11 & 11,0 \\
\hline Não & 89 & 89,0 \\
\hline Total & 100 & 100,0 \\
\hline \multicolumn{3}{|l|}{ Consultas pré-natal } \\
\hline Nenhuma & 61 & 61,0 \\
\hline $1-3$ & 37 & 37,0 \\
\hline 4 ou mais & 2 & 2,0 \\
\hline Total & 100 & 100,0 \\
\hline \multicolumn{3}{|l|}{ Idade na época da menarca (anos) } \\
\hline Até 10 & 2 & 2,0 \\
\hline $11-12$ & 36 & 36,0 \\
\hline 13 ou mais & 62 & 62,0 \\
\hline \multirow{2}{*}{\multicolumn{3}{|c|}{ Idade na época da $1^{1}$ relação sexual (anos) }} \\
\hline & & \\
\hline Até 14 & 10 & 10,0 \\
\hline 15 a 18 & 75 & 75,0 \\
\hline 19 ou mais & 15 & 15,0 \\
\hline Total & 100 & 100,0 \\
\hline \multicolumn{3}{|l|}{ Número de parceiros sexuais } \\
\hline Um & 28 & 28,0 \\
\hline Mais de um & 72 & 72,0 \\
\hline Total & 100 & 100,0 \\
\hline \multicolumn{3}{|l|}{ Doenças prévias } \\
\hline Infecção urinária & 1 & 1,0 \\
\hline Hipertensão & 2 & 2,0 \\
\hline Mioma & 3 & 3,0 \\
\hline Ovários policísticos & 2 & 2,0 \\
\hline Nenhuma & 92 & 92,0 \\
\hline Total & 100 & 100,0 \\
\hline \multicolumn{3}{|l|}{ Abortamento prévio } \\
\hline Sim & 18 & 18,0 \\
\hline Não & 82 & 82,0 \\
\hline Total & 100 & 100,0 \\
\hline
\end{tabular}


Tabela 4. Características do abortamento sofrido pelas mulheres internadas. São Luís, Maranhão, 2015.

\begin{tabular}{|c|c|c|}
\hline Variáveis & $\mathbf{n}$ & $\%$ \\
\hline \multicolumn{3}{|l|}{ Tipo } \\
\hline Espontâneo & 88 & 88,0 \\
\hline Provocado & 12 & 12,0 \\
\hline Total & 100 & 100,0 \\
\hline \multicolumn{3}{|l|}{ Causa referida do abortamento } \\
\hline Descolamento de placenta & 1 & 1,0 \\
\hline Gravidez embrionária & 4 & 4,0 \\
\hline Gravidez tubária & 1 & 1,0 \\
\hline Malformação fetal & 1 & 1,0 \\
\hline Uso de misoprostol & 5 & 5,0 \\
\hline Queda & 7 & 7,0 \\
\hline Não referida & 81 & 81,0 \\
\hline Total & 100 & 100,0 \\
\hline \multicolumn{3}{|l|}{ Método do abortamento no hospital } \\
\hline Curetagem & 100 & 100,0 \\
\hline Outros & 0 & 0,0 \\
\hline Total & 100 & 100,0 \\
\hline \multicolumn{3}{|c|}{ Idade gestacional na data do abortamento } \\
\hline Até 12 semanas & 82 & 82,0 \\
\hline Mais de 12 semanas & 14 & 14,0 \\
\hline Não referida & 4 & 4,0 \\
\hline Total & 100 & 100,0 \\
\hline
\end{tabular}

não referiram causa. $\mathrm{Na}$ maternidade, o método da curetagem foi feito em $100,0 \%$ das pacientes estudadas. A idade gestacional na data do abortamento foi de até 12 semanas em $82,0 \%$ dos casos.

\section{Discussão}

O registro de $57,0 \%$ de mulheres na faixa etária de 20 a 28 anos foi maior que o encontrado por pesquisadores em uma Maternidade Escola do Recife (48,9\%), que estudaram 160 mulheres no período de 2005 a $2006^{14}$. No entanto, foi semelhante a um estudo sobre abortamento em uma favela de São Paulo, que mostrou a média de idade de 23,1 anos $^{15}$.
A cor parda encontrada em pouco mais da metade dos casos foi referida pelas pacientes, sendo assim um dado subjetivo, não permitindo fazer inferências. Numa pesquisa na favela de São Paulo foram encontrados $52,6 \%$ de mulheres pardas/mulatas, corroborando o presente estudo ${ }^{15}$.

A situação de mulheres casadas de $28,0 \%$, somadas a de mulheres em união estável no presente estudo é menor que o encontrado no estudo realizado pela Maternidade Escola na Cidade do Recife, que encontrou $90,1 \%$ de mulheres com companheiros fixos ${ }^{14}$. Compreende-se que a procedência de $81,0 \%$ das mulheres seja de São Luís devido à localização da Maternidade Benedito Leite nesta capital. 
Mais da metade das pacientes estudadas possuíam ensino médio completo ou chegaram até o nível superior. Contudo, houve discordância de uma pesquisa em capitais brasileiras no ano de 2012 sobre aborto ilegal que mostrou maior frequência entre mulheres de escolaridade mais baixa $(39,0 \%)^{16}$.O Ministério da Saúde, em 2009, descreveu o perfil educacional dessas mulheres mostrando aumento no número de anos na escola, mas a maioria com até 8 anos de estudo ${ }^{8}$.

$A$ renda mensal referida pelas pacientes estudadas de $62,0 \%$ de 2 a 3 salários mínimos coincidiu com outro trabalho realizado na mesma cidade envolvendo maternidades públicas não específicas, onde 0 mesmo apresentou uma maioria de $57,5 \%$ de mulheres com renda entre 1 a 3 salários mínimos ${ }^{17}$.A renda familiar de até 3 salários mínimos, faz parte do perfil de mulheres que sofreram abortamento descrito pelo Ministério da Saúde em 2009 sobre 20 anos do aborto e saúde pública no Brasil $^{8}$.

Quanto à ocupação, mulheres com trabalho remunerado superaram em pouco as pacientes que têm como ocupação atividades do lar, diferindo de estudos que apresentaram a situação contrária onde mais da metade correspondem a atividades do lar $(56,82 \%)^{18}$. Esse perfil de mulheres do lar, no entanto, veio mudando ao longo dos anos, corroborando com o encontrado no presente trabalho ${ }^{8}$.

O presente estudo não evidenciou o uso do fumo entre as mulheres avaliadas, o que corrobora com o estudo feito em um hospital universitário de Porto Alegre, RS, no ano de 2008, que estudou fatores associados ao tabagismo na gestação e apontou uma minoria de fumantes $(19,1 \%)^{19}$.

O alcoolismo foi um fator de risco encontrado entre as mulheres estudadas, mostrando um registro de $11,0 \%$ de ingestão durante a gestação, enquanto o estudo sobre - consumo de bebida alcoólica entre gestantes em um ambulatório de pré-natal em um hospital universitário do estado do Rio de Janeiro, no ano de 2006, mostrou um registro de $10 \%{ }^{20}$.

A maioria das pacientes no presente estudo teve poucas consultas pré-natal, sendo 98,0\% com menos de 4 consultas. $O$ estudo sobre fatores associados ao uso do álcool e cigarro realizado em uma maternidade pública do Rio de Janeiro entre 1999 e 2006 mostrou uma média de 8,2 consultas $^{21}$.

A idade de menarca de 13 anos ou mais em $62,0 \%$ das mulheres foi próxima do encontrado em outro estudo. Quanto à sexarca, o resultado foi de $75,0 \%$ para idades entre 15 e 18 anos. No estudo sobre 0 aborto entre adolescentes atendidas em uma maternidade pública de Teresina, o resultado para menarca encontrado foi de 12 - 13 em $66,0 \%$ e o da sexarca foi de $95,0 \%$ para menores de 16 anos $^{22}$.

O número de abortos foi maior em mulheres com mais parceiros sexuais $(72,0 \%)$, assim como no estudo realizado sobre a vulnerabilidade de mulheres jovens à violência sexual e aborto, nos anos de 2001 e 2002, em Salvador, Rio de Janeiro e Porto Alegre, que evidenciou $86,2 \%{ }^{23}$.

Em relação a abortamentos prévios, foi encontrado a porcentagem de $82,0 \%$ de mulheres que referiram 
não ter tido abortamento anterior, resultado próximo ao relatado em outro estudo que mostrou o registro de $75,4 \%$ das mulheres referindo a mesma situação ${ }^{24}$.

Em relação ao tipo de abortamento, houve concordância com outros estudos, mostrando uma maioria de abortos do tipo espontâneo $(88,0 \%)^{25}$.A maioria das pacientes (81,0\%) não referiu causa. Desta forma, sendo um dado referido não permite fazer inferências. $\mathrm{O}$ método da curetagem, feito em 100,0\% das pacientes estudadas, corresponde ao procedimento mais frequente em abortamentos dentro de maternidades ${ }^{26}$.A idade gestacional na data do abortamento teve sua maioria em abortos que ocorreram em até 12 semanas, correspondendo a $82,0 \%$ dos casos; esse valor coincide com a maioria dos abortamentos descritos na literatura $^{11}$.

\section{Conclusão}

Concluiu-se que as mulheres em situação de abortamento na Maternidade Benedito Leite, em São Luís - MA encontram-se na faixa etária jovem, em união estável e com condições socioeconômicas precárias. Houve poucos fatores de risco, mas a maioria não fez consulta pré-natal.A maioria referiu aborto espontâneo e fez curetagem na maternidade.

O abortamento constitui-se num importante problema de saúde pública no Brasil. Políticas públicas direcionadas para esta problemática devem se fazer presentes nas mulheres desde a escola, com uma educação voltada para a prevenção de gravidez indesejada, a inserção da mulher no mercado de trabalho, o aconselhamento e a informação adequados para o planejamento familiar e o acompanhamento prénatal precoce. Desta forma o presente trabalho espera ter contribuído com informações relevantes para os gestores públicos atuarem na promoção da saúde da mulher.

\section{Referências}

1. FEBRASGO. Federação Brasileira das Associações de Ginecologia e Obstetrícia. Manual de Orientação: Assistência ao Abortamento, Parto e Puerpério. 2010. [acesso 2015 abr4]. Disponível em: http://professor.ucg.br/SiteDocente ladmin/arquivosUpload/13162/mat erial/ASSIST\%C3\%8ANCIA\%20A O\%20PARTO,\%20PUERP\%C3\%8 9RIO\%20E\%20ABORTAMENTO\% 20-\%20FEBRASGO\%202010.pdf

2. Pinto AP; Tocci HA. O aborto provocado e suas consequências. Rev Enferm UNISA 2003; 4:56-61. [acesso 2015 abr4]. Disponível em:

http://www.unisa.br/graduacao/biol ogicas/enfer/revista/arquivos/200313.pdf.

3. Sarmento D. Legalização do Aborto e Constituição. Mundo Jurídico, 2005. [acesso 2015 mar 18]. Disponível em: http://www.mundojuridico.adv.br.

4. Brasil. Lei n. 2848 de 7 de dezembro de 1940. Dos crimes contra a pessoa. Dos crimes 
contra a vida. Código Penal. Art.128. Diário Oficial da República Federativa do Brasil, Brasília, DF, 31 dez. 1940.

5. Ribeiro GG, Alencar CA. Maternidade-Escola Assis Chateaubriand. Protocolos de Conduta - Obstetrícia. 2013. [acesso 2015 mar 18]. Disponível em:

http://www.meac.ufc.br/arquivos/bi blioteca cientifica/File/PROTOCO LO\%200BSTETRICIA/obstetriciaa bril2013/obstetriciacap1.pdf

6. Copeland, L. Tratado de Ginecologia. Editorial Médica Panamericana, 2002.

7. WHO. World Health Organization. Complications of abortion: technical and managerial guidelines for prevention and treatment. Geneva: World Health Organization; 1995. [acesso 2015 mar 28]. Disponívelem: http://whqlibdoc.who.int/publication s/1995/9241544694.pdf

7. Brasil. Ministério da Saúde. Secretaria de Ciência, Tecnologia e Insumos Estratégicos. Departamento de Ciência e Tecnologia. Aborto e saúde pública no Brasil: 20 anos / Ministério da Saúde, Secretaria de Ciência, Tecnologia e Insumos Estratégicos, Departamento de Ciência e Tecnologia. - Brasília: Ministério da Saúde, 2009. [acesso 2015 mar 15]. Disponível em: http://bvsms.saude.gov.br/bvs/publ icacoes/livro aborto.pdf.

8. Valongueiro S. Mortalidade materna por aborto: fontes, métodos e instrumentos de estimação. Anais do $12^{\circ}$ Encontro
Nacional de Estudos Populacionais. [acesso 2015 mar 16]. Disponível em: http://www.abep.nepo.unicamp.br/ docs/anais/pdf/2000/Todos/Mortali dade\%20\%28materna\%29\%20por $\% 20$ Aborto\%20-

\%20Fontes,\%20M\%C3\%A9todos.. ..pdf

9. Laurenti R; Jorge MHPM; Gotlieb SLD. A mortalidade materna nas capitais brasileiras: algumas características e estimativa de um fator de ajuste. Rev. Bras. Epidemiol. v. 7, n 4, 2004. [acesso 2015 abr3]. Disponível em: http://www.scielo.br/pdf/rbepid/v7n 4/08.pdf.

10. Cunningham FG, Leveno $\mathrm{KJ}$, Bloom SL, Spong CY, Dashe JS, Hoffman BL, Casey BM, Sheffield JS. Williams Obstetrics. 24 edição, McGraw-Hill, 2014.

11. Barini R; Sarno M; Couto E; Zaccaria R; Cavalcante M; Silva JLP. Revisão sobre as Diferentes Etiologias no Aborto Espontâneo Recorrente. Femina, v. 34 n. 8, p. 533-6, 2006. [acesso 2015 abr4]. Disponível em: http://www.febrasgo.org.br/site/wpcontent/uploads/2013/05/Femina 34-8-533.pdf

12. MS. Ministério da Saúde. Secretaria de Atenção à Saúde. Departamento de Ações Programáticas Estratégicas. Gestação de alto risco: manual técnico. 5. ed. - Brasília: Editora do Ministério da Saúde, 2012.

13. Ramos KS, Ferreira ALCG, Souza AI. Mulheres hospitalizadas 
DOI: https://doi.org/10.24863/rib.v7i1.16

em uma Maternidade Escola na

Cidade do Recife, Brasil. RevEscEnferm USP 2010; 44(3):605-10.

14. Fusco CLB, Andreoni S, Silva RS. Epidemiologia do aborto inseguro em uma população em situação de pobreza - Favela Inajar de Souza, São Paulo. RevBrasEpidemiol 2008; 11(1): 7888.

15. Diniz D, Medeiros M.Itinerários e métodos do aborto ilegal em cinco capitais brasileiras. Ciência \& Saúde Coletiva, Rio de Janeiro, v. 17, n. 7, 2012.

16. Araújo MCR, Mochel EG. Aborto provocado: fatores associados em mulheres admitidas em maternidades públicas em São Luís - MA, 2006. Rev. paul. enferm;27(2):[79-86], abr.- jun. 2008. tab.

17. Domingos SRF, Merighi MAB, Faria ECR, Ferreira LMG. Características dos abortamentos de mulheres atendidas em uma instituição hospitalar filantrópica de Caratinga - MG. Rev. Min. Enferm.;15(4): 504-512, out./dez., 2011.

18. Motta GCP, Echer IC, Lucena AF. Fatores associados ao tabagismo na gestação. Rev. Latino-Am. Enfermagem.18(4):[08 telas] jul-ago 2010.

19. Oliveira TR, Simões SMF. O consumo de bebida alcoólica pelas gestantes: um estudo exploratório. Esc Anna Nery RevEnferm2007 dez; 11 (4): 632 - 8.
20. Freire K, Padilha PC, Saunders

C. Fatores associados ao uso de álcool e cigarro na gestação. RevBrasGinecol Obstet. 2009; 31(7):335-41.

21. Monteiro Esoh, Silva IP, Sousa SS. Perfil socioeconômico e epidemiológico do aborto entre adolescentes atendidas em uma maternidade pública de Teresina. R. Interd. v. 8, n. 1, p. 194-203, jan. fev. mar. 2015.

22. Pilecco FB. Aborto e violência sexual: 0 contexto de vulnerabilidade entre mulheres e jovens. 2010. 141f. [Mestrado]. Universidade Federal do Rio Grande do Sul. 2010.

23. Borges A, Correia T, Abrantes A. Perfil das mulheres que sofreram abortamento espontâneo. XVI Encontro Nacional da Associação Portuguesa dos Enfermeiros Obstetras. Livro de Resumos. Aveiro. p. 41-42. 2013.

24. Botelho NM, Araújo SG, Souza DC. Aspectos clínicoepidemiológicos das mulheres pós-abortamento em hospital de referência. 2008. [Trabalho de conclusão de curso]. Universidade Federal do Pará.

25. Brasil. Atenção Humanizada ao

Abortamento: norma

técnica/Ministério da Saúde,

Secretaria de Atenção à Saúde,

Departamento de Ações

Programáticas Estratégicas -

Brasília: Ministério da Saúde, 2005. 